\title{
REMARKS ON FIXED POINT THEOREMS AND THEIR EXTENSIONS
}

\author{
BY \\ W. V. PETRYSHYN( $\left.{ }^{1}\right)$
}

1. Introduction. Let $X$ be a real or complex Banach space and let $C$ be a closed bounded convex subset of $X$. If $S$ is a completely continuous $\left({ }^{2}\right)$ mapping of $C$ into $C$, then according to the Schauder fixed point principle (i.e., Theorem 1 in [14]) $S$ has a fixed point in $C$. In [6], [7] Kačurovskir, using a variational principle, has extended Schauder fixed point principle to yield the existence of solutions in certain balls $B_{r}$ of a Hilbert space $H$ or a Banach space $X$ for the nonlinear equation of the form

$$
T(x)=S(x),
$$

where $S$ is a completely continuous and $T(x)$ is essentially a strongly monotone $\left({ }^{2}\right)$ potential mapping. On the other hand, utilizing the properties of the degree of a mapping, $\mathrm{Al}^{\prime}$ tman [2] extended in another direction Schauder's (as well as Rothe's [13] and Krasnosel'skil's [8]) fixed point principles by proving the existence of fixed points for completely continuous mappings $S$ of $B_{r}$ into $X$ which on the boundary $\partial B_{r}$ of $B_{r}$ satisfy the condition

$$
\|S(x)-x\|^{2} \geqq\|S(x)\|^{2}-\|x\|^{2}, \quad x \in \partial B_{r} .
$$

In his recent paper, Shinbrot [15], apparently unaware of $\mathrm{Al}^{\prime}$ tman's paper [1], proved the existence of fixed points for weakly continuous $\left({ }^{2}\right)$ mappings $S$ of $B_{r}$ into $H$ which on $\partial B_{r}(\subset H)$ satisfy the condition

$$
\operatorname{Re}(S x, x) \leqq\|x\|^{2}, \quad x \in \partial B_{r} .
$$

The purpose of this note is twofold. First, using the recent extensive results of Browder [3], [4], [5], Minty [9], [10], and Zarantonello [17], and others [11] concerning the monotone and complex monotone $\left({ }^{2}\right)$ operators, we extend and strengthen in $\$ 2$ the assertions in [6], [7] by generalizing the fixed point principles in [1], [14], [15] to yield solutions of equation (i) in which the operator $S$ is assumed to be either completely continuous, strongly continuous $\left({ }^{2}\right)$, or even weakly continuous from a subset of a reflexive Banach space $X$ into its conjugate space $X^{*}$ while the operator $T$ is assumed to be either complex monotone or to satisfy a certain weaker condition, the so-called, $k$-condition $\left({ }^{2}\right)$, as a mapping from $X$ to $X^{*}$.

Presented to the Society, August 3, 1965; received by the editors June 10, 1965.

(1) The author is grateful to the referee for his valuable suggestions.

$\left({ }^{2}\right)$ For the precise definitions of the notions used in the Introduction and the statements of the corresponding results see $\$ 2$. 
Second, in $\$ 3$ we give a simple proof of $\mathrm{Al}^{\prime}$ tman's [2] fixed point theorem without the use of the notion of the degree of a mapping. As a byproduct, we obtain the theorems of Rothe [13] and Krasnosel'skiĩ [8].

Finally let us add that the problem of how one actually constructs fixed points of certain mappings, if one knows their existence, is considered in [12].

2. Generalizations of fixed point theorems. Let $X$ be a complex reflexive Banach space and let $X^{*}$ be its conjugate space considered as the space of bounded conjugate linear functionals on $X$. For $f$ in $X^{*}$ and $x$ in $X$ let us denote the value of $f$ at $x$ by $(f, x)$. Let $T$ be a (not necessarily linear) operator from $X$ to $X^{*}$. To avoid any possible confusion of terms we shall define here some of the notions to be used in the sequel. Following [2], [4], [9], [16], [17] we say that $T$ is continuous if $x_{n} \rightarrow x$ strongly in $X$ implies $T x_{n} \rightarrow T x$ strongly in $X^{*} ; T$ is demicontinuous if $x_{n} \rightarrow x$ strongly in $X$ implies $T x_{n} \rightarrow T x$ weakly in $X^{*} ; T$ is strongly continuous if $x_{n} \rightarrow x$ weakly in $X$ implies $T x_{n} \rightarrow T x$ strongly in $X^{*} ; T$ is weakly continuous if $x_{n} \rightarrow x$ weakly in $X$ implies $T x_{n} \rightarrow T x$ weakly in $X^{*} ; T$ is compact if $T$ maps every bounded set in $X$ onto a precompact set in $X^{*} ; T$ is completely continuous if $T$ is continuous and compact; $T$ is demiclosed if $x_{n} \rightarrow x$ strongly in $X$ and $T x_{n} \rightarrow u$ weakly in $X^{*}$ imply that $T x=u ; T$ is locally bounded if $\left\{T x_{n}\right\}$ is bounded in $X^{*}$ whenever $\left\{x_{n}\right\}$ is a Cauchy sequence in $X ; T$ is monotone if for all $x$ and $y$ in $X$

$$
\operatorname{Re}(T x-T y, x-y) \geqq 0
$$

and strongly monotone if for all $x$ and $y$ in $X$ and some $\alpha>0$

$$
\operatorname{Re}(T x-T y, x-y) \geqq \alpha\|x-y\|^{2} .
$$

Let us note in passing that the strong continuity of $T$ implies its complete continuity (as well as its weak continuity) but, as we shall see later, in general the converse is not true. However, the following lemma which we shall utilize below is valid.

LEMMA 1. If $T$ is compact and weakly continuous, then $T$ is strongly continuous.

Proof. Let $\left\{x_{n}\right\}$ be any sequence in $X$ with $x_{n} \rightarrow x$ weakly in $X$ and suppose that $T x_{n} \nrightarrow T x$ strongly in $X^{*}$. Then there exists a subsequence $\left\{T x_{n_{t}}\right\}$, with $n_{i} \rightarrow \infty$, and some positive number $\delta>0$ such that

$$
\left\|T x_{n_{i}}-T x\right\| \geqq \delta .
$$

Since $\left\{T x_{n_{i}}\right\} \subset\left\{T x_{n}\right\}$ and $\left\{T x_{n}\right\}$ is compact there is a subsequence $\left\{T x_{k_{i}}\right\}$ such that $T x_{k_{i}} \rightarrow u$ strongly in $X^{*}$. This and the assumed weak continuity of $T$ (as a mapping from $X$ to $X^{*}$ ) and the reflexivity of $X$ imply that for every $y$ in $X$ we have

$$
\lim \left(T x_{n}, y\right)=(T x, y) \text { and } \lim \left(T x_{k_{i}}, y\right)=(u, y) .
$$

Hence $(T x, y)=(u, y)$ for every $y$ in $X$. This implies that $T x=u$. Consequently

$$
\lim _{i}\left\|T x_{k_{i}}-T x\right\|=0 .
$$


The last equality contradicts the inequality (2) and thus shows that the supposition $T x_{n} \nrightarrow T x$ strongly in $X^{*}$ is false.

In this section we show in a simple manner that, in conjunction with the recent extensive results of Browder [3], [4], [5], Minty [9], [10], Zarantonello [17], Shinbrot [15], and others [1], [11], Schauder's Theorem 1 and Theorem 2 in [14] can be generalized to yield the existence of solutions in certain balls of $X$ for the nonlinear equations of the form

$$
T(x)=S(x)
$$

where $S$ is either completely continuous, strongly continuous, or even weakly continuous and $T$ satisfies certain conditions to be specified below. At the same time we obtain the extension of results stated by Kačurovskir [6], [7].

THEOREM 1. Let $X$ be a complex reflexive Banach space and let $T(x)$ be a mapping from $X$ to $X^{*}$ such that $T$ is demicontinuous and

$$
|(T x-T y, x-y)| \geqq \beta\|x-y\|^{2}
$$

for all $x$ and $y$ in $X$ and some constant $\beta>0$ (operators $T$ for which (4) holds will be referred to as complex monotone). Let $S(x)$ be a completely continuous mapping such that $(1 / \beta)\{S(x)-T(o)\}$ maps the ball $B_{r} \equiv\{x \in X:\|x\| \leqq r, r>0\}$ into the ball $B_{r^{*}}^{*} \equiv\left\{u \in X^{*}:\|u\| \leqq r^{*}, r^{*}>0\right\}$ with $r^{*} \leqq r$. Then there exists at least one point $x_{0} \in B_{r}$ such that

$$
T\left(x_{0}\right)=S\left(x_{0}\right)
$$

Proof. Since $T$ is a demicontinuous mapping of $X$ into $X^{*}$ which satisfies the inequality (4), Zarantonello-Browder's Theorem [4], [17] assures us that $T$ is a one-to-one mapping of $X$ onto $X^{*}$ with a continuous inverse $T^{-1}$ defined on all of $X^{*}$. Moreover, (4) implies that $T^{-1}$ is a Lipschitzian mapping with constant $1 / \beta$ since, by putting $u=T x$ and $v=T y$ and using the Schwarz-Bunjakovskir inequality, we obtain from (4) the relation

$$
\left\|T^{-1} u-T^{-1} v\right\| \leqq(1 / \beta)\|u-v\|
$$

which is valid for all $u$ and $v$ in $X^{*}$.

Let $y$ be an arbitrary but fixed element in $B_{r}$. The above discussion implies that there exists a unique $x$ in $X$ such that $T x=S y$. Furthermore, for each $y$ in $B_{r}$, the corresponding $x=T^{-1}(S y)$ belongs to $B_{r}$ since, in view of our assumption and (4), $(1 / \beta)\{T x-T(o)\}=(1 / \beta)\{S y-T(o)\}$ belongs to $B_{r}^{*}$ and

$$
\|x\|^{2} \leqq|((1 / \beta)\{T x-T(o)\}, x)|=|((1 / \beta)\{S y-T(o)\}, x)| \leqq r^{*}\|x\| \leqq r\|x\| .
$$

This implies that $x=T^{-1}(S y) \in B_{r}$. Thus the operator $T^{-1} S$ maps $B_{r}$ into $B_{r}$. Moreover, if in (6) we put $u=S y$ and $v=S z$ with $y$ and $z$ in $B_{r}$, then

$$
\left\|T^{-1} S y-T^{-1} S z\right\| \leqq(1 / \beta)\|S y-S z\|
$$


from which we easily deduce the complete continuity of the operator $T^{-1} S$. In fact, the continuity of $T^{-1} S$ follows immediately from (7) and the continuity of $S$ while the compactness of the set $T^{-1} S\left(B_{r}\right)$ in $X$ follows from (7) and the compactness of the set $S\left(B_{r}\right)$ in $X^{*}$. Indeed, the complete continuity of $S$ implies that for any given $\varepsilon>0$ there exists a finite number of elements $\left\{S u_{1}, \ldots, S u_{n}\right\}$ forming a $\beta \varepsilon$-net for the set $S B_{r}$ and, therefore, (7) shows that $\left\{T^{-1} S u_{1}, \ldots, T^{-1} S u_{n}\right\}$ forms an $\varepsilon$-net for the set $T^{-1} S B_{r}$.

Since $T^{-1} S$ is a completely continuous operator from $B_{r}$ to $B_{r}$, Schauder's Theorem 1 [14] assures us that $T^{-1} S$ has a fixed point in $B_{r}$, i.e., there exists at least one point $x_{0} \in B_{r}$ such that (5) holds.

REMARK $1\left(^{3}\right)$. Theorem 1 a fortiori remains valid when $T$ is a demicontinuous strongly monotone operator from $X$ to $X^{*}$. It is easy to see that for the latter class of operators our Theorem 1 is also true for real Banach spaces. In case $X$ is a real Banach space and $T$ is a strongly monotone and so-called potential operator (and hence continuous), Theorem 1 was stated without proof by Kačurovskil [7]. Let us add that $T$ is called potential on $X$ if there exists a functional $f$ such that $\operatorname{grad} f(x)=T(x)$ for every $x$ in $X$.

Consider now the case when $S$ is strongly continuous. Here the assertion of Theorem 1 above holds for $T$ satisfying somewhat less stringent conditions.

In what follows, the operator $T$ will be called $X^{*}$-demi-invertible if $T^{-1}$ exists and is a demicontinuous mapping from $X^{*}$ to $X . T$ will be said to satisfy the $k$ condition if $T$ is $X^{*}$-demi-invertible and there exists a constant $k>0$ such that for all $x$ in $X$

$$
|(T x-T(o), x)| \geqq k\|x\|^{2} .
$$

$T$ will be called strongly closed if $x_{n} \rightarrow x$ weakly in $X$ and $T x_{n} \rightarrow u$ strongly in $X^{*}$ imply that $T x=u$.

It follows from the Minty-Browder Theorem [3], [9] and the ZarantonelloBrowder Theorem that demicontinuous strongly monotone and complex monotone operators satisfy the $k$-condition.

LEMMA 2. If $T$ is a one-to-one strongly closed mapping of $X$ onto $X^{*}$ such that $T^{-1}$ is locally bounded and (8) holds, then $T$ satisfies the $k$-condition.

Proof. We need only to show that $T^{-1}$ is demicontinuous as a mapping from $X^{*}$ to $X$, i.e., if $v_{n} \rightarrow v$ strongly in $X^{*}$ then $T^{-1} v_{n} \rightarrow T^{-1} v$ weakly in $X$. To prove the demicontinuity of $T$ assume to the contrary that $v_{n} \rightarrow v$ strongly in $X^{*}$ and $T^{-1} v_{n} \nrightarrow T^{-1} v$ weakly in $X$. Then there exists a unit element $f$ in $X^{*}$, a constant $\varepsilon>0$ and a subsequence $\left\{v_{n_{k}}\right\}$ such that

$$
\left|\left(f, T v_{n_{k}}^{-1}-T^{-1} v\right)\right| \geqq \varepsilon .
$$

${ }^{(3)}$ For the connection between our Theorem 1 and those of Browder [18] see Remark 4 below. 
Since $v_{n_{k}} \rightarrow v$ strongly in $X^{*}$ and $T^{-1}$ is locally bounded, $\left\{T v_{n_{k}}^{-1}\right\}$ is a bounded sequence in a reflexive space $X$. Hence there exists a subsequence $\left\{T v_{n_{m}}^{-1}\right\}$ of $\left\{T v_{n_{k}}^{-1}\right\}$ and some element $x$ in $X$ such that $x_{n_{m}} \equiv T^{-1} v_{n_{m}} \rightarrow x$ weakly in $X$. But then $T x_{n_{m}}=v_{n_{m}} \rightarrow v$ strongly in $X^{*}$ and therefore, since $T$ is strongly closed, $v=T x$. Consequently,

$$
\left(f, T v_{n_{m}}^{-1}-T v^{-1}\right) \rightarrow 0,
$$

which contradicts the inequality $\left(^{*}\right)$. This contradiction shows that $\left\{T^{-1} v_{n}\right\}$ converges weakly to $T^{-1} v$, i.e., $T^{-1}$ is demicontinuous.

In case $X$ is a separable Hilbert space $H$ the following lemma is also valid.

LEMMA 3. (a) The class of weakly continuous strongly monotone operators $T$ from $H$ into $H$ satisfies the $k$-condition.

(b) The class of all $T$ such that $T$ is a one-to-one mapping of the form $T x=c x+F x$ (where $c>0$ and $F$ is a weakly continuous mapping on $H$ such that $\operatorname{Re}(F(t x), x)$ is a monotonically increasing function on $0 \leqq t \leqq 1$ for every $x$ in $H$ ) and $T^{-1}$ is locally bounded satisfies the $k$-condition.

(c) The class of all operators $T$ of the form $T=I-A$ with

$$
\|A x-A y\| \leqq l\|x-y\|, \quad 0<l<1, \quad x, y \in H,
$$

satisfies the $k$-condition.

Proof. (a) follows from the results of Shinbrot [15] who proved a stronger assertion for the mappings of the form $T(x) \equiv \alpha x+F(x)$, where $F$ is monotone and $\alpha>0$. However, if $T$ satisfies the inequality $\left(1_{0}\right)$ above, then the mapping $F(x)$ $\equiv T(x)-\alpha x$ is clearly monotone and, conversely, since

$$
(F x-F y, x-y)=(T x-T y, x-y)-\alpha(x-y, x-y) \geqq \alpha\|x-y\|^{2}-\alpha\|x-y\|^{2}=0 .
$$

Moreover, $T(x)$ and $T(x)=\alpha x+F(x)$ have the same behavior with respect to the properties specified in (a). Thus, (a) is true.

(b) Let us first observe that, since $\operatorname{Re}(F(t x), x)$ is a monotonically increasing function of $t$ in $[0,1]$, we have for all $x$ in $H$

$$
\operatorname{Re}(F(o), x) \leqq \operatorname{Re}(F x, x) \text { or } \operatorname{Re}(F(o)-F x, x) \leqq 0 .
$$

This shows that $T x=c x+F x$ satisfies the inequality (8) with $k=c$ since

$$
\operatorname{Re}(T x-T(o), x)=\operatorname{Re}(c x+F x-F(o), x)=c\|x\|^{2}+\operatorname{Re}(F x-F(o), x) \geqq c\|x\|^{2}
$$

and $|(T x-T(o), x)| \geqq \operatorname{Re}(T x-T(o), x)$.

Furthermore, it was shown in [15] that, under present conditions, $T$ maps $H$ onto $H$. Hence $T^{-1}$ is defined on all of $H$. Moreover, $T^{-1}$ is demicontinuous because if $x_{n} \rightarrow x$ strongly in $H$, then our conditions imply that $T^{-1} x_{n} \rightarrow T^{-1} x$ weakly in $H$. Indeed, in virtue of the local boundedness of $T^{-1},\left\{T^{-1} x_{n}\right\}$ is bounded and therefore there is a subsequence $\left\{T^{-1} x_{n_{i}}\right\}$ such that $T^{-1} x_{n_{i}} \rightarrow u$ weakly in $H$. On the 
other hand, $x_{n_{i}} \rightarrow x$ strongly in $H$ (and hence weakly in $H$ ) and $x_{n_{i}}=T\left(T^{-1} x_{n_{i}}\right)$ $\rightarrow T u$ weakly in $H$ since $T$ is weakly continuous. Hence $x=T u$ or $u=T^{-1} x$. This implies the demicontinuity of $T^{-1}$.

(c) The last assertion follows from the fact that, in view of our condition, $T$ is continuous and strongly monotone.

THEOREM 2. Let $T$ be an operator from $X$ to $X^{*}$ which satisfies the $k$-condition and let $S$ be a strongly continuous operator from the ball $B_{r}$ to $X^{*}$ such that $(1 / k)\{S(x)-T(o)\}$ maps $B_{r}$ into $B_{r}^{*}$ with $r^{*} \leqq r$. Then there exists at least one element $x_{0} \in B_{r}$ such that

$$
T\left(x_{0}\right)=S\left(x_{0}\right)
$$

Proof. The proof of Theorem 2 is similar to the proof of Theorem 1. As before we show that, since $T$ satisfies the $k$-condition, for each $y$ in $B_{r}$ the corresponding solution $x=T^{-1}(S y)$ belongs to $B_{r}$, i.e., $T^{-1} S$ maps $B_{r}$ into $B_{r}$. Furthermore, $T^{-1} S$ is weakly continuous as a mapping from $B_{r}$ into $B_{r}$. Indeed, if $\left\{x_{n}\right\}$ is a sequence from $B_{r}$ such that $x_{n} \rightarrow x$ weakly in $X$, then $x \in B_{r}$ and $S\left(x_{n}\right) \rightarrow S(x)$ strongly in $X^{*}$ since $B_{r}$ is weakly closed and $S$ is strongly continuous as a mapping from $B_{r}$ to $X^{*}$. Since $T$ satisfies the $k$-condition (and hence $T^{-1}$ is demicontinuous as a mapping from $X^{*}$ to $\left.X\right) T^{-1} S x_{n} \rightarrow T^{-1} S x \in B_{r}$ weakly in $X$. Therefore, Schauder's Theorem 2 [14] assures us that $T^{-1} S$ has a fixed point in $B_{r}$, i.e., equation (9) has at least one solution $x_{0}$ in $B r$.

In case $X$ is a separable Hilbert space $H$ a slightly stronger version of Theorem 2 is valid.

THEOREM 3. Let $T$ be an operator from $H$ to $H^{*}$ which satisfies the $k$-condition and let $S$ be a strongly continuous operator from the ball $B_{r}$ to $H$ such that $(1 / k)\{S(x)-T(o)\}$ maps the boundary $\partial B_{r}$ of $B_{r}$ into $B_{r}$. Then there exists at least one point $x_{0} \in B_{r}$ such that $T\left(x_{0}\right)=S\left(x_{0}\right)$.

Proof. It follows from our conditions that $T^{-1} S$ maps $B_{r}$ into $H$ and $\partial B_{r}$ into $B_{r}$. To verify the last assertion, let $\tilde{y}$ be an arbitrary element in $\partial B_{r}$ and let $\tilde{x}=T^{-1}(S \tilde{y})$ be the corresponding solution of $T x=S \tilde{y}$. Then, by our assumption, the element $(1 / k)\{T \tilde{x}-T(o)\}=(1 / k)\{S \tilde{y}-T(o)\}$ belongs to $B_{r}$ and, consequently, the $k$-condition of $T$ implies that for every $\tilde{y} \in B_{r}$

$$
\|\tilde{x}\|^{2}=\left\|T^{-1}(S \tilde{y})\right\|^{2} \leqq|((1 / k)\{S \tilde{y}-T(o), \tilde{x})\}| \leqq r\|\tilde{x}\|=r\left\|T^{-1}(S \tilde{y})\right\|
$$

which shows that $T^{-1} S$ maps $\partial B_{r}$ into $B_{r}$. Finally, $T^{-1} S$ is a weakly continuous mapping from $B_{r}$ to $H$ and for every $\tilde{y} \in \partial B_{r}$

$$
\operatorname{Re}\left(\tilde{y}, T^{-1} S \tilde{y}\right) \leqq\left|\left(\tilde{y}, T^{-1} S \tilde{y}\right)\right| \leqq\left\|T^{-1} S \tilde{y}\right\|\|\tilde{y}\| \leqq r\|\tilde{y}\|=\|\tilde{y}\|^{2} .
$$

Thus, by Theorem 2 of Shinbrot [15], $T^{-1} S$ has at least one fixed point $x_{0}$ in $B_{r}$, i.e., there exists a point $x_{0} \in B_{r}$ such that $T\left(x_{0}\right)=S\left(x_{0}\right)$. 
REMARK 2. Let us observe that if in this case we assume that $S$ is only weakly continuous, then the assertion of Theorem 3 remains valid provided only that in the definition of the $k$-condition of $T$ the condition that $T^{-1}$ be demicontinuous be replaced by the requirement that $T^{-1}$ be weakly continuous.

SPECIAL CASES. Let us observe that the theorems we proved above were obtained for spaces and operators satisfying rather general conditions. It is therefore reasonable to expect that by specializing these spaces and the operators $T$ and $S$ we obtain a number of particular theorems and among them many of the known ones.

We shall illustrate our observation by showing that practically all fixed point theorems stated by Kačurovskir [6], [7] for more restrictive class of operators are special cases of our theorems.

(i) If in our Theorem 1 we let $X$ be a real Hilbert space $H$ and $T$ be a strongly monotone operator with $\alpha \geqq 1$, then Theorem 1 yields the validity of Theorem 4 in [7] without the additional assumption that $\|T x-T y\| \leqq m\|x-y\|$.

On the other hand, if as in [7, Theorem 5] $F$ is assumed to be a potential operator on $H$ and $A$ to be a mapping on $H$ such that

$$
(F x-F y, x-y) \leqq a_{1}\|x-y\|^{2}, \quad\|A x-A y\| \leqq b\|x-y\|,
$$

where $0 \leqq b<1-a_{1}$, then by taking $X=H$ and $T=I-F-A$ we see that $T$, so defined, is a strongly monotone mapping on $H$ with $\alpha=1-a_{1}-b>0$. Furthermore, $T$ is demicontinuous since $F$, being a potential mapping, is continuous and so is $A$. The other condition is equivalent to our condition. Consequently, our Theorem 1 applies.

(ii) Similar comment applies to Theorem 1 in [6] for less restrictive operators $F$ if we take $T=I-F$ and observe that other conditions there certainly imply our weaker conditions.

Finally, if, as in [6, Theorem 1], the potential operator $F$ satisfies the inequality (10) with $a_{1}+1<0$ and $\|F(o)\| \leqq-\left(1+a_{1}\right) r / 2$ and $S(x)$ is a completely continuous mapping from $B_{r}$ into itself, then $T$ defined by $T \equiv I-F$ is a continuous strongly monotone operator with $\alpha>1-a_{1}>1$ and

$$
\left\|\frac{1}{\alpha}\{S x-T(o)\}\right\|=\left\|\frac{1}{\alpha}\{S x+F(o)\}\right\| \leqq \frac{1}{\alpha}(\|S x\|+\|F(o)\|) \leqq \frac{r}{1-a_{1}}-\frac{1+a_{1}}{2\left(1-a_{1}\right)} r=\frac{r}{2},
$$

i.e., $(1 / \alpha)\{S x-T(o)\}$ maps $B_{r}$ into itself. Consequently, our Theorem 1 is applicable.

RemarK 3. Let us remark in passing that if $H$ is a separable Hilbert space and $B_{r}$ is a closed ball in $H$ for any $r>0$, then there exists a completely continuous mapping $S$ from $B_{r}$ into $B_{r}$ such that $S$ is not weakly continuous on $B_{r}$. We shall illustrate our assertion by the following simple example.

EXAmple. We let $H=L_{2}(0,1), K(s, t)$ a uniformly continuous function of its arguments on the square $R: 0 \leqq s, t \leqq 1$ such that $0<m \leqq K(s, t) \leqq 1 / r$ on $R$ for an 
arbitrary, but fixed, $r>0$. For each $x(t)$ in $B_{r}$ we define

$$
S(x)=\int_{0}^{1} K(s, t) x^{2}(t) d t .
$$

Since, in view of our condition, for every $x(t)$ in $B_{r}$

$$
\|S(x)\|^{2}=\int_{0}^{1}\left\{\int_{0}^{1} K(s, t) x^{2}(t) d t\right\}^{2} d s \leqq\{1 / r\}^{2} r^{4}=r^{2},
$$

it follows that $S$ maps $B_{r}$ into itself. Furthermore, as was shown in [16, pp. 14-15] $S(x)$ is completely continuous on $B_{r}$. Now, in virtue of our condition on $K(s, t)$, it follows that for any $x(t) \in \partial B_{r}$

$$
\|S(x)\|=\left[\int_{0}^{1}\left\{\int_{0}^{1} K(s, t) x^{2}(t) d t\right\}^{2} d s\right]^{1 / 2} \geqq m r^{2}>0 .
$$

The inequality (12) and Lemma 1 show that $S(x)$ cannot be weakly continuous on $B_{r}$ for the assumption that $S(x)$ is weakly continuous leads to the contradiction of (12). Indeed, assuming that $S(x)$ is weakly continuous, we see that for any sequence $\left\{x_{n}(t)\right\}$, with $x_{n} \in \partial B_{r}$, which converges weakly to 0 the sequence $\left\{S\left(x_{n}\right)\right\}$ converges also weakly to $S(o)=0$. On the other hand, since $S(x)$ is completely continuous (and hence compact), Lemma 1 shows that $S\left(x_{n}\right)$ converges strongly to $S(o)=0$. This is, however, the contradiction of (12). Thus $S(x)$ is not weakly continuous on $B_{r}$.

Finally, let us observe that Remark 3 seems to invalidate Shinbrot's assertion that his result (Theorem 2 in [15]) includes the Schauder fixed point principle for Hilbert spaces at least when the complete continuity of an operator is understood in the sense of the definition stated at the beginning of this paper, which, in fact, was the definition employed by Schauder [14]. His assertion remains valid, however, for strongly continuous operators which have precisely the property used by Shinbrot [15, p. 258] and to which, unfortunately, many authors refer to as a complete continuity property. (I.e., a number of authors call an operator $T$ completely continuous if $T x_{n} \rightarrow T x$ strongly whenever $x_{n} \rightarrow x$ weakly.)

3. Simple proof of $\mathbf{A l}^{\prime}$ tman's fixed point theorem. In this section we give a simple proof of Al'tman's [2] fixed point theorem for a completely continuous operator in $X$ without the use of the notion of the degree of a mapping in the sense of Leray-Schauder or the use of the homotopy theory. As a byproduct, we obtain the theorems of Rothe [13], Krasnosel'skii [8] for the case of a Hilbert space, and others.

THEOREM 4 (AL'TMAN). If $S$ is a completely continuous mapping from $B_{r}$ to $X$ such that for every $x \in \partial B_{r}$

$$
\|x-S x\|^{2} \geqq\|S x\|^{2}-\|x\|^{2}, \quad x \in \partial B_{r},
$$

then $S$ has at least one fixed point in $B_{r}$. 
Proof. Let $R$ be the retraction map of $X$ on $B_{r}$ given by

$$
\begin{aligned}
R u & =u & & \text { if }\|u\| \leqq r, \\
& =r u /\|u\| & & \text { if }\|u\| \geqq r .
\end{aligned}
$$

If for all $x$ in $B_{r}$ we define the mapping $S_{1}(x)$ by $S_{1}(x) \equiv R S x$, then it follows from the definition of $R$ and the complete continuity of $S$ that $S_{1}$ is a completely continuous mapping of $B_{r}$ into $B_{r}$. Hence, by Schauder's Theorem 1 [14], $S_{1}$ has at least one fixed point $x_{0}$ in $B_{r}$ so that $S_{1} x_{0}=x_{0}$. But then $x_{0}$ is also a fixed point of $S$. Indeed, since $x_{0} \in B_{r}$, then either $\left\|x_{0}\right\|<r$ or $\left\|x_{0}\right\|=r$ and

$$
\begin{aligned}
x_{0}=S_{1} x_{0}=R S x_{0} & =S x_{0} & & \text { if }\left\|S x_{0}\right\| \leqq r, \\
& =r S x_{0} /\left\|S x_{0}\right\| & & \text { if }\left\|S x_{0}\right\| \geqq r .
\end{aligned}
$$

Thus, it is sufficient to discuss the case when $\left\|S x_{0}\right\| \geqq r$, i.e., the equation

$$
S x_{0}=\lambda_{0} x_{0}, \quad \lambda_{0}=\left\|S x_{0}\right\| / r, \quad\left(\left\|S x_{0}\right\| \geqq r\right),
$$

since in the other case $x_{0}=R s x_{0}=S x_{0}$ and $x_{0}$ is a fixed point of $S$.

Case 1. If $\left\|x_{0}\right\|<r$ and $\left\|S x_{0}\right\| \geqq r$, then the equality (15) cannot hold since it would lead to the contradiction of the inequality $\left\|x_{0}\right\|<r$.

Case 2. If $\left\|x_{0}\right\|=r$ and $\left\|S x_{0}\right\| \geqq r$, then $\lambda_{0} \geqq 1$ and the assumption (15) leads to the following equalities:

$$
\left\|x_{0}-S x_{0}\right\|^{2}=\left\|x_{0}-\lambda_{0} x_{0}\right\|^{2}=\left(1-\lambda_{0}\right)^{2} r
$$

and

$$
\left\|S x_{0}\right\|^{2}-\left\|x_{0}\right\|^{2}=\lambda_{0}^{2}\left\|x_{0}\right\|^{2}-\left\|x_{0}\right\|^{2}=\left(\lambda_{0}^{2}-1\right) r .
$$

Since $\lambda_{0} \geqq 1$, the above equalities contradict the condition (13) unless $\lambda_{0}=1$, i.e., unless $x_{0}$ is a fixed point of $S$. This completes the simple proof of $\mathrm{Al}^{\prime}$ tman's theorem.

SPECIAL CASES. (i) Suppose that instead of (13) we assume that for all $x \in \partial B_{r}$

$$
\|S x\| \leqq\|x\|, \quad x \in \partial B_{r} .
$$

Then, by (16), for all $x$ in $\partial B_{r}$

$$
\begin{aligned}
\|x-S x\|^{2} & \geqq(\|x\|-\|S x\|)^{2}=\|x\|^{2}-2\|x\|\|S x\|+\|S x\|^{2} \\
& \geqq\|x\|^{2}-2\|x\|^{2}+\|S x\|^{2}=\|S x\|^{2}-\|x\|^{2}
\end{aligned}
$$

and, consequently, we obtain a simple proof of the Rothe Theorem [13] as a special case of Theorem 4.

(ii) A similar remark holds if instead of (13) we assume that $S$ satisfies the stronger condition

$$
\|x-S x\| \geqq\|S x\|, \quad x \in \partial B_{r} .
$$

(iii) If $X$ is now a real Hilbert space $H$, then as was pointed out by $\mathrm{Al}^{\prime}$ tman, the 
condition (13) is equivalent in this case to the condition

$$
(S x, x) \leqq\|x\|^{2}, \quad x \in \partial B_{r},
$$

imposed by Krasnosel'skii [8]. Hence we obtain also a simple proof of Krasnosel'skiř's theorem for Hilbert spaces as a special case of Theorem 4.

REMARK 4. After this paper had been written the author received from F. E. Browder a preliminary draft of his paper [18] in which, it seems, $\$ 2$ presents a generalization of our Theorem 1 (i.e., when Theorem 1 is specialized to the case when $X$ is a real space, $T$ is demicontinuous and strongly monotone, and $S$ is strongly continuous) to the class of finitely continuous and semimonotone operators $\tilde{T}(x)$ of $X$ into $X^{*}$, where $\tilde{T}$ is said to be finitely continuous if it is continuous from each finite dimensional subspace of $X$ to the weak topology of $X^{*}$ and $T$ is semimonotone if it is obtained from a mapping $f$ of $X \times X$ into $X^{*}$ (i.e., $\tilde{T}(x) \equiv f(x, x)$ ) such that $f(x, y)$ is monotone in $x$ for fixed $y$ and strongly continuous in $y$ for fixed $x$.

It is easy to see that if in our case we define the mapping $f(x, y)$ by $f(x, y)$ $\equiv A x-S y$, then $\tilde{T}(x) \equiv T x-S x$ is finitely continuous and semimonotone since the finite continuity of $\tilde{T}$ follows from the demicontinuity of $T$ and strong continuity of $S$ while its semimonotonicity follows from the monotonicity of $T$ and the strong continuity of $S$.

It should be emphasized, however, that in both cases (our case of Theorem 1 for the class of operators of the form $\tilde{T}(x)=T x-S x$ and Browder's theorems for semimonotone operators $\tilde{T}(x))$ the operator $\tilde{T}(x)$ consists essentially of two parts: a monotone part satisfying some mild continuity conditions and a strongly continuous part.

Added in proof. If $X$ is a real Banach space with a Schauder basis, then in virtue of the author's very recent results in [19] on projectionally-compact (or $P$-compact) operators, Theorems 1, 2, and 3 remain valid if instead of complete continuity of $S$ in Theorem 1 or of weak continuity of $S$ in Theorems 2 and 3 we assume that $S$ is such that the operator $T^{-1} S$ is $P$-compact. Theorem 4 remains also valid if $S$ is assumed to be only $P$-compact and its proof does not employ Schauder's fixed point theorem.

\section{REFERENCES}

1. M. Al'tman, A fixed point theorem in Hilbert space, Bull. Acad. Polon. Sci. Cl. III 5 (1957), 19-22.

2. - , A fixed point theorem in Banach space, Bull. Acad. Polon. Sci. Cl. III 5 (1957), 89-92.

3. F. E. Browder, Nonlinear elliptic boundary value problems, Bull. Amer. Math. Soc. 69 (1963), 862-874.

4. - Remarks on nonlinear functional equations, Proc. Nat. Acad. Sci. U.S.A. 51 (1964), 985-989.

5. - Remarks on nonlinear functional equations. II, Illinois J. Math. 9 (1965), 608-616. 
6. R. I. Kačurovskir̆, On some fixed point principles, Ucen. Zap. Moskov. Reg. Ped. Inst. 96 (1960), 215-219.

7. - On monotone operators and convex functionals, Uspehi Mat. Nauk 15 (1960), 213-215.

8. M. A. Krasnosel'skir, New existence theorems for solutions of nonlinear integral equations, Dokl. Akad. Nauk SSSR 88 (1953), 949-952.

9. G. J. Minty, Monotone (nonlinear) operators in Hilbert space, Duke Math. J. 29 (1962), 341-346.

10. —_ On a "monotonicity" method for the solution of nonlinear equations in Banach space, Proc. Nat. Acad. Sci. U.S.A. 50 (1963), 1038-1041.

11. W. V. Petryshyn, On the extension and the solution of nonlinear operator equations, Illinois J. Math. 10 (1966), 255-274.

12. - Construction of fixed points of demicompact mappings in Hilbert space, J. Math. Anal. Appl. 14 (1966), 276-284.

13. E. Rothe, Zur Theorie der topologischen Ordnung und der Vektorfelder in Banachschen Räumen, Compositio Math. 5 (1937), 177-197.

14. J. Schauder, Der Fixpunktsatz in Funktionalräumen, Studia Math. 2 (1930), 171-180.

15. M. Shinbrot, $A$ fixed point theorem and some applications, Arch. Rational Mech. Anal. 17 (1964), 255-271.

16. M. M. Vainnberg, Variational methods for the study of nonlinear operators, Holden-Day, San Francisco, Calif., 1964. (Translated from Russian by A. Feinstein.)

17. E. Zarantonello, The closure of the numerical range contains the spectrum, Bull. Amer. Math. Soc. 7 (1964), 781-787.

18. F. E. Browder, Mapping theorems for noncompact nonlinear operators in Banach spaces, Proc. Nat. Acad. Sci. U.S.A. 54 (1965), 337-342.

19. W. V. Petryshyn, On non-linear P-compact operators in Banach space with applications to constructive fixed point theorems, J. Math. Anal. Appl. 15 (1966), 228-242.

UNIVERSITY OF CHICAGo,

Chicago, Illinois 